\title{
THE DUST EMISSIVITY SPECTRAL INDEX IN THE STARLESS CORE TMC-1C
}

\author{
Scott Schnee ${ }^{1,8}$, Melissa Enoch ${ }^{2}$, Alberto Noriega-Crespo ${ }^{3}, \mathrm{Jack}_{\text {Sayers }}{ }^{4}$, Susan Terebey $^{5}$, Padla Caselli $^{6}$, \\ Jonathan Foster ${ }^{7}$, Alyssa Goodman ${ }^{7}$, Jens Kauffmann $^{7}$, Deborah PadgetT ${ }^{3}$, Luisa Rebull $^{3}$, Anneila Sargent ${ }^{1}$, \\ AND RAHUl SHETTY ${ }^{7}$ \\ ${ }^{1}$ Department of Astronomy, California Institute of Technology, MC 105-24 Pasadena, CA 91125, USA; scott.schnee@nrc-cnrc.gc.ca \\ ${ }^{2}$ Department of Astronomy, University of California, Berkeley, CA 94720, USA \\ ${ }^{3}$ Spitzer Science Center, MC 220-6, California Institute of Technology, Pasadena, CA 91125, USA \\ ${ }^{4}$ Jet Propulsion Laboratory, California Institute of Technology, 4800 Oak Grove Drive, Pasadena, CA 9110, USA \\ ${ }^{5}$ Department of Physics and Astronomy PS315, 5151 State University Drive, California State University at Los Angeles, Los Angeles, CA 90032, USA \\ ${ }^{6}$ School of Physics and Astronomy, University of Leeds, Leeds LS2 9JT, UK \\ ${ }^{7}$ Harvard-Smithsonian Center for Astrophysics, 60 Garden Street, Cambridge, MA 02138, USA \\ Received 2009 July 27; accepted 2009 November 6; published 2009 December 8
}

\begin{abstract}
In this paper, we present a dust emission map of the starless core TMC-1C taken at $2100 \mu \mathrm{m}$. Along with maps at $160,450,850$, and $1200 \mu \mathrm{m}$, we study the dust emissivity spectral index from the (sub)millimeter spectral energy distribution, and find that it is close to the typically assumed value of $\beta=2$. We also map the dust temperature and column density in TMC-1C, and find that at the position of the dust peak $\left(A_{V} \sim 50\right)$ the line-of-sight-averaged temperature is $\sim 7 \mathrm{~K}$. Employing simple Monte Carlo modeling, we show that the data are consistent with a constant value for the emissivity spectral index over the whole map of TMC-1C.
\end{abstract}

Key words: dust, extinction - stars: formation

Online-only material: color figures

\section{INTRODUCTION}

Starless cores are often identified through maps of their dust emission at (sub)millimeter wavelengths, and recent surveys of nearby molecular clouds have increased the number of such objects considerably (e.g., Hatchell et al. 2007; Enoch et al. 2008; Kauffmann et al. 2008; Simpson et al. 2008). Analysis of the properties of starless cores is often restricted by sparse sampling of their spectral energy distribution (SED), uncertain dust emissivities, and assumptions about their geometries. As a result, the measured (sub)millimeter fluxes are often used in conjunction with assumed values for the dust temperature and/or emissivity to determine core masses, though the systematic errors can be substantial (a factor of a few). With sufficient spectral coverage and knowledge of source geometry, it should be possible to map the column density, temperature, and dust emissivity spectral index in a starless core, though this has yet to be accomplished.

One of the best-studied starless cores is TMC-1C, located in the Taurus molecular cloud at an approximate distance of $140 \mathrm{pc}$ (Torres et al. 2009). Previous studies of the dust emission from TMC-1C at 450, 850, and $1200 \mu \mathrm{m}$ have determined that it is cold $\left(T_{d} \sim 6 \mathrm{~K}\right)$ and dense $\left(n \sim 10^{6} \mathrm{~cm}^{-3}\right)$ at its center, and becomes progressively less dense and warmer at larger radii (Schnee et al. 2007b; Schnee \& Goodman 2005). The dustderived mass of TMC-1C is a factor of a few larger than its virial mass, and self-absorbed $\mathrm{N}_{2} \mathrm{H}^{+}$spectra show evidence for sub-sonic infall (Schnee et al. 2007a; Schnee \& Goodman 2005). The chemistry of TMC-1C is characterized by the depletion of $\mathrm{CO}$ and its isotopologues, as well as depletion of $\mathrm{CS}$ and $\mathrm{N}_{2} \mathrm{H}^{+}$ (Schnee et al. 2007a).

Previously reported mass and temperature profiles for TMC$1 \mathrm{C}$ are estimated assuming a constant value for the emissivity spectral index ( $\beta=1.8$; Schnee et al. 2007b). However, a wide range of values for $\beta$ in other regions have been measured or

\footnotetext{
8 Current address: NRC-HIA, 5071 West Saanich Road, Victoria, BC V9E 2E7, Canada.
}

calculated on theoretical grounds. For instance, in the diffuse interstellar medium (ISM), an emissivity spectral index of $\beta=2$ fits the typical far-infrared spectrum well (Draine et al. 2007; Draine \& Lee 1984), while grain growth in protostellar disks results in values of $\beta \simeq 1$ (Beckwith \& Sargent 1991). Since starless cores are in an intermediate stage between the diffuse ISM and protostars, one might expect that the dust in a starless core would have an emissivity spectral index in the range $1 \leqslant \beta \leqslant 2$, although the timescale of grain growth at densities $n_{H} \sim 10^{6} \mathrm{~cm}^{-3}$ is several hundred times the free-fall time of a typical starless core (Chakrabarti \& McKee 2005). On the other hand, the growth of icy mantles on dust grains could steepen the slope of the dust SED, and this has been cited as the reason for high values of the emissivity spectral index in parts of the Orion Ridge ( $\beta \sim 2.5$; Lis et al. 1998) and in Sgr B2(N) $(\beta \sim 3.7$; Kuan et al. 1996). In the cold and dense environment in TMC$1 \mathrm{C}$, the dust grains could form similar icy mantles, which might drive the emissivity spectral index to values $\beta>2$.

In addition to changes in $\beta$, grain growth by either coagulation or accretion of an icy mantle will change the opacity of the dust grains, $\kappa$. For instance, evidence that dust opacities are higher in dense cores than in the diffuse ISM can be found by comparing gas temperature and dust emission profiles (Keto \& Caselli 2008) or by comparing dust and gas emission profiles (Keto et al. 2004). From models of dust coagulation for conditions that are plausible for a starless core, Ossenkopf \& Henning (1994) show that the dust opacity at $1.3 \mathrm{~mm}$ can be a factor of 5 higher than in a diffuse environment. Using $160 \mu \mathrm{m}$ data for Taurus, Terebey et al. (2009) measure an opacity of $\kappa_{160 \mu \mathrm{m}}=0.23 \pm 0.046 \mathrm{~cm}^{2} \mathrm{~g}^{-1}$ in the cold cloud $\left(A_{V}\right.$ ranging from 0.4 to 4.0 ). The opacity is 2.6 times greater than the diffuse ISM opacity (Weingartner \& Draine 2003), and provides evidence for higher opacity values in dense regions (compared to Ossenkopf \& Henning 1994). However, a study of the dust properties of a filament in the Taurus Molecular Ring, located close to TMC-1C, has found no evidence for grain coagulation and instead finds uniform grain properties (Nutter et al. 2008). 
Laboratory studies of possible dust grain materials have measured the dust emission and absorption properties at far-infrared and longer wavelengths. For instance, Aannestad (1975) measured the absorption of silicate grains and found that $\beta \simeq 2$ for grains without an ice mantle, and $\beta \simeq 3$ for grains with an ice mantle. The steepest spectral indices calculated by Aannestad (1975) were for fused quartz and olivine with ice mantles, both of which had $\beta=3.5$, close to the extremely steep $\beta=3.7$ reported by Kuan et al. (1996). In a study of the millimeter-wave absorption of silicate grains in the temperature range $1.2-30 \mathrm{~K}$, Agladze et al. (1996) found that the emissivity spectral index depends on the dust temperature and for some materials can vary between $1.5 \leqslant \beta \leqslant 2.5$, with the largest value for $\beta$ at a temperature $T_{d} \sim 10 \mathrm{~K}$. A temperature-dependent emissivity spectral index was also found by Mennella et al. (1998) for cosmic dust analog grains in the temperature range $24 \mathrm{~K} \leqslant T_{d} \leqslant 295 \mathrm{~K}$ and a wavelength range $20 \mu \mathrm{m} \leqslant \lambda \leqslant 2 \mathrm{~mm}$, with a steeper SED at lower temperatures. The range of emissivity spectral indices is $1.2 \leqslant \beta \leqslant 2.4$ for the various grain types considered by Mennella et al. (1998). In a study of amorphous silicates in the temperature range $10 \mathrm{~K} \leqslant T_{d} \leqslant 300 \mathrm{~K}$ and wavelength range $0.1 \mathrm{~mm} \leqslant \lambda \leqslant 2 \mathrm{~mm}$, Boudet et al. (2005) also report an anticorrelation between $T_{d}$ and $\beta$, with values of the emissivity spectral index between $1.5 \leqslant \beta \leqslant 2.5$. Based on these laboratory measurements, one might expect that in starless cores like TMC-1C, which have temperatures close to $10 \mathrm{~K}$ (Schnee et al. 2009), the emissivity spectral index would be close to $\beta \simeq 2.5$.

Observations of nearby star-forming regions suggest an anticorrelation between $T_{d}$ and $\beta$ similar to that measured in the laboratory. For instance, observations of the Orion, $\rho$ Ophiuchi and Taurus molecular clouds at far-infrared and (sub)millimeter wavelengths by Dupac et al. (2003) show that the emissivity spectral index may vary from $2.5 \geqslant \beta \geqslant 1.0$ as the dust temperature rises from $10 \mathrm{~K} \leqslant T_{d} \leqslant 80 \mathrm{~K}$. Similarly, in a study of the far-infrared and (sub)millimeter SEDs of luminous infrared galaxies, Yang \& Phillips (2007) report that the emissivity spectral index varies from $2.4 \geqslant \beta \geqslant 0.9$ as the dust temperature varies from $30 \mathrm{~K} \leqslant T_{d} \leqslant 60 \mathrm{~K}$. In a study of the starless core L1498, Shirley et al. (2005) find an emissivity spectral index of $\beta=2.44 \pm 0.62$ for an assumed dust temperature of $T_{d}=10.5 \pm 0.5 \mathrm{~K}$, consistent with the hypothesis that the cold dust in a starless core has a steep spectral index.

However, variations in the dust properties along the line of sight and noise in the observed maps can mimic an anticorrelation between $T_{d}$ and $\beta$ (Shetty et al. 2009a, 2009b). Furthermore, at high densities and low temperatures, grain coagulation will decrease $\beta$ while the possible $T_{d}-\beta$ relation will increase the emissivity spectral index. Therefore, it is not at all clear that one should expect the emissivity spectral index to vary significantly in a starless core. Observations of dust emission and extinction in the dark cloud B68 show no evidence for variations in the dust properties (Bianchi et al. 2003), suggesting that to some extent the various pressures on $\beta$ may cancel out.

In this paper, we present maps of the starless core TMC-1C at $160,450,850,1200$, and $2100 \mu \mathrm{m}$. By spatially resolving the emission from TMC-1C over a wide range of wavelengths, we then map the column density, temperature, and emissivity spectral index of the dust, which has typically been difficult to do with previous data sets.

\section{OBSERVATIONS}

The 160, 450, 850, and $1200 \mu \mathrm{m}$ maps have been presented or described in previous papers (Terebey et al. 2009; Schnee
\& Goodman 2005; Schnee et al. 2007b; Di Francesco et al. 2008; Kauffmann et al. 2008), so here we briefly review the observations and data reduction, and refer the reader to the earlier papers for more details. The $2100 \mu \mathrm{m}$ map has not been previously presented, so it is discussed in detail below.

\subsection{Spitzer}

The Spitzer data near TMC-1C derive from the Taurus Spitzer Legacy Survey (D. L. Padgett et al. 2010, in preparation), a large $44 \mathrm{deg}^{2}$ map of the Taurus star-forming region at seven wavelengths ranging from 3.6 to $160 \mu \mathrm{m}$. See D. L. Padgett et al. (2010, in preparation) for an overview of the survey parameters and data processing. The MIPS data utilize the fast scan mode with $3 \mathrm{~s}$ integration time per frame and five visits per pixel. The MIPS $160 \mu \mathrm{m}$ data obtained in fast scan mode mapping does not have enough redundancy to fill in completely all the data gaps due to a dead readout and other effects. To mitigate these data gaps and to preserve the diffuse emission as much as possible, a 3 pixel by 3 pixel ( $15^{\prime \prime}$ per pixel) median filter is applied to the image. The median filter introduces some image smoothing, increasing the effective angular resolution from $40^{\prime \prime}$ to $1^{\prime}$. In some cases, filtering reduces the surface brightness by $10 \%-15 \%$, though the reduction in the map of $\mathrm{TMC}-1 \mathrm{C}$ is only $2 \%$.

Analysis of the $160 \mu \mathrm{m}$ data in Taurus shows there are two main cloud components: emission from the extended cold cloud $\left(14.2 \pm 0.4 \mathrm{~K}, A_{V}<3\right)$, and emission from compact cold cores ( $T_{d} \sim 10 \mathrm{~K}$, high $A_{V}$; Terebey et al. 2009; Flagey et al. 2009). Since our present focus is the high extinction regions sampled by (chopped) millimeter data, here we further process the $160 \mu \mathrm{m}$ data to remove the spatially extended cloud emission. Following the procedure in Terebey et al. (2009), we use IRAS $100 \mu \mathrm{m}$ images to model and subtract the $14.2 \mathrm{~K}$ cloud emission. The resulting $160 \mu \mathrm{m}$ image traces the high extinction ridge and looks very similar to the millimeter continuum emission. The median flux removed from original $160 \mu \mathrm{m}$ map is $96 \mathrm{MJy} \mathrm{sr}^{-1}$, or about $90 \%$ of the total flux. Uncertainties in the temperature of the extended cloud component results in a $10 \%$ uncertainty in the final $160 \mu \mathrm{m}$ map. Given the $12 \%$ absolute flux uncertainty of MIPS $160 \mu \mathrm{m}$ maps (Stansberry et al. 2007), we conservatively estimate the absolute uncertainty in the $160 \mu \mathrm{m}$ "cold core" map to be $20 \%$.

\subsection{SCUBA}

Although in previous papers on TMC-1C, we used submillimeter maps observed and reduced by us (Schnee \& Goodman 2005; Schnee et al. 2007b), in this paper we use maps of TMC$1 \mathrm{C}$ at 450 and $850 \mu \mathrm{m}$ from the new SCUBA Legacy Catalogues (Di Francesco et al. 2008). We choose to use the new maps because they include all of the data taken with SCUBA (Holland et al. 1999) at all epochs and use the most current calibration and reduction methods. The noise in the maps are 132 and $21 \mathrm{mJy} \mathrm{beam}^{-1}$ at 450 and $850 \mu \mathrm{m}$, respectively, and have resolutions of approximately $9^{\prime \prime}$ and $14^{\prime \prime}$. The uncertainties in the absolute flux calibration (primarily due to beam-shape uncertainty and fluctuations of opacity above the telescope during observations and calibration), are $20 \%$ at $850 \mu \mathrm{m}$ and $50 \%$ at $450 \mu \mathrm{m}$, as described in Di Francesco et al. (2008). We set the zero point of the Legacy maps by determining the median flux density in two regions of the core but nearby and subtracting these values $\left(-0.32\right.$ and $-0.035 \mathrm{Jy}$ beam $\left.^{-1}\right)$ from the 450 and $850 \mu \mathrm{m}$ maps. 


\section{3. $M A M B O$}

We observed the $1200 \mu \mathrm{m}$ continuum emission from TMC$1 \mathrm{C}$ in the autumns of 2002 and 2003 using the MAMBO-2 array (Kreysa et al. 1999) on the IRAM $30 \mathrm{~m}$ telescope on Pico Veleta (Spain), producing a map with 10 '.7 resolution. Data were taken with on-the-fly mapping, with the telescope subreflector chopping in azimuth by $60^{\prime \prime}-70^{\prime \prime}$, and the resultant image was reconstructed using the Emerson et al. (1979) algorithm iteratively to properly reproduce large-scale emission. The noise in map is $3 \mathrm{mJy}_{\text {beam }}^{-1}$, and the flux calibration uncertainty, as derived from the rms of calibrator observations across pooled observing sessions and the uncertainty in the intrinsic calibrator fluxes, is about $10 \%$. For further details on the $1200 \mu \mathrm{m}$ observations and data reduction, see Schnee et al. (2007b) and Kauffmann et al. (2008).

\subsection{Bolocam}

We observed the $2100 \mu \mathrm{m}$ continuum emission from TMC$1 \mathrm{C}$ in the autumn of 2007 using Bolocam on the Caltech Submillimeter Observatory (CSO). Data were collected in raster scan mode at a scan speed of $240^{\prime \prime} \mathrm{s}^{-1}$, with a step size between rasters of $11^{\prime \prime}$. Each raster was $20^{\prime}$ in length, and each of the 105 total scans were separated by $11^{\prime \prime}$. The resultant map has a coverage of $\sim 20^{\prime} \times 20^{\prime}$ with $60^{\prime \prime}$ resolution. Three sets of observations contain rasters parallel to right ascension, and the other three contain rasters parallel to declination. Data reduction and flux calibration, accomplished through observations of Uranus and Neptune, was done as described in Sayers et al. (2009).

Observations at approximately $2 \mathrm{~mm}$ are dominated by sky noise. Although the Bolocam pipeline principal component analysis (PCA) cleaning algorithm efficiently removes sky noise, it also removes some of the source flux. This decreases the peak intensity, introduces negative bowls around bright sources, and reduces the brightness on scales larger than $1^{\prime}$ for extended sources. We recover this lost flux using a mapping algorithm that iteratively subtracts a source model from the real data (for more description and performance evaluation, see Enoch et al. 2006). This technique is similar to CLEAN (Högbom 1974; Schwarz 1978), but works in the image plane, and the real data are used for the source model rather than, e.g., a Gaussian component model. The first source model is formed from the original map, including only regions with signal-to-noise ratio greater than 1.5. That source model is subtracted from the original timestream data, which is then re-cleaned and mapped without the source flux. This process is iterated until the source model stabilizes, 10 iterations in this case. TMC-1C is a faint source, so the correction is relatively small, with the source flux density increasing by up to $30 \%$ after iterative mapping. The noise is the $2100 \mu \mathrm{m}$ map is $8 \mathrm{mJy}$ beam $^{-1}$, and the uncertainty in the absolute flux calibration is $\sim 20 \%$.

\section{ANALYSIS}

Here we estimate the temperature, column density, and emissivity spectral index in TMC-1C using our flux density maps at $160,450,850,1200$, and $2100 \mu \mathrm{m}$. Under the assumptions that the dust is isothermal and characterized by a single emissivity spectral index, the flux density per beam in each map can be described as a modified blackbody, given by

$$
S_{v}=\Omega B_{v}\left(T_{d}\right) \kappa_{v} \mu m_{H} N_{\mathrm{H}_{2}}
$$

where

$$
B_{v}\left(T_{d}\right)=\frac{2 h v^{3}}{c^{2}} \frac{1}{\exp \left(h v / k T_{d}\right)-1}
$$

and

$$
\kappa_{v}=\kappa_{230}\left(\frac{v}{230 \mathrm{GHz}}\right)^{\beta} .
$$

In Equation (1), $S_{v}$ is the flux density per beam, $\Omega$ is the solid angle of the beam, $B_{v}\left(T_{d}\right)$ is the blackbody emission from the dust at temperature $T_{d}, \mu=2.8$ is the mean molecular weight of interstellar material in a molecular cloud per hydrogen molecule, $m_{H}$ is the mass of the hydrogen atom, $N_{\mathrm{H}_{2}}$ is the column density of hydrogen molecules, and a gas-to-dust ratio of 100 is assumed. In Equation (3), $\kappa_{230}=0.009 \mathrm{~cm}^{2} \mathrm{~g}^{-1}$ is the emissivity of the dust grains at a gas density of $10^{6} \mathrm{~cm}^{-3}$ covered by a thin ice mantle at $230 \mathrm{GHz}$ (Ossenkopf \& Henning 1994, Column 6 of Table 1), and $\beta$ is the emissivity spectral index of the dust. Although Equations (1)-(3) are often used to described the observed emission from starless cores, such observations average over a range of dust grain properties. Furthermore, the emissivity $\left(\kappa_{230}\right)$ is uncertain by roughly a factor of 2 . If we had chosen a different value for $\kappa_{230}$, this would affect the derived column density, but would have no impact on the derived temperature and emissivity spectral index. We do not consider variations in $\kappa_{230}$ in this paper, but instead save this analysis for a future analysis.

\subsection{Color Correction}

The fluxes given in the dust emission maps are derived from the total energy detected convolved with the wavelengthdependent instrumental response and an assumed SED of the emitting source. The assumed SEDs in the Spitzer, SCUBA, MAMBO, and Bolocam maps are that of a 10,000 K blackbody, a flat-spectrum source, a flat-spectrum source, and a blackbody in the Rayleigh-Jeans regime, respectively. Because we are observing emission from dust, with an assumed SED of a modified blackbody, the quoted flux at the nominal wavelength of each map ought to be corrected. However, the relatively narrow bandpasses of the detectors results in a modest correction factor.

For each map, we use the instrumental response and our bestguess dust SED of a modified blackbody with $T_{d}=10 \mathrm{~K}$ and $\beta=2$ to find the "reference" wavelength of the observations. The integral of the convolution of the instrumental response and the dust SED are equal above and below this reference wavelength:

$$
\int_{0}^{\lambda_{\text {ref }}} S_{\lambda} R(\lambda) d \lambda=\int_{\lambda_{\text {ref }}}^{\infty} S_{\lambda} R(\lambda) d \lambda,
$$

where $S_{\lambda}$ is the flux emitted by the dust at wavelength $\lambda$ and $R(\lambda)$ is the instrumental response at wavelength $\lambda$.

The reference wavelength, used in our calculations of the dust emission properties, are close to the nominal wavelength of each map, with the biggest difference in the MAMBO map because of its wide bandpass. In all maps, the flux uncertainties are larger than the correction from using the reference wavelength in our calculations rather than the nominal wavelength. The derived reference wavelengths are given in Table 1.

\subsection{Single $S E D$}

One way to estimate the dust temperature, column density and emissivity spectral index is to fit modified blackbody SEDs 

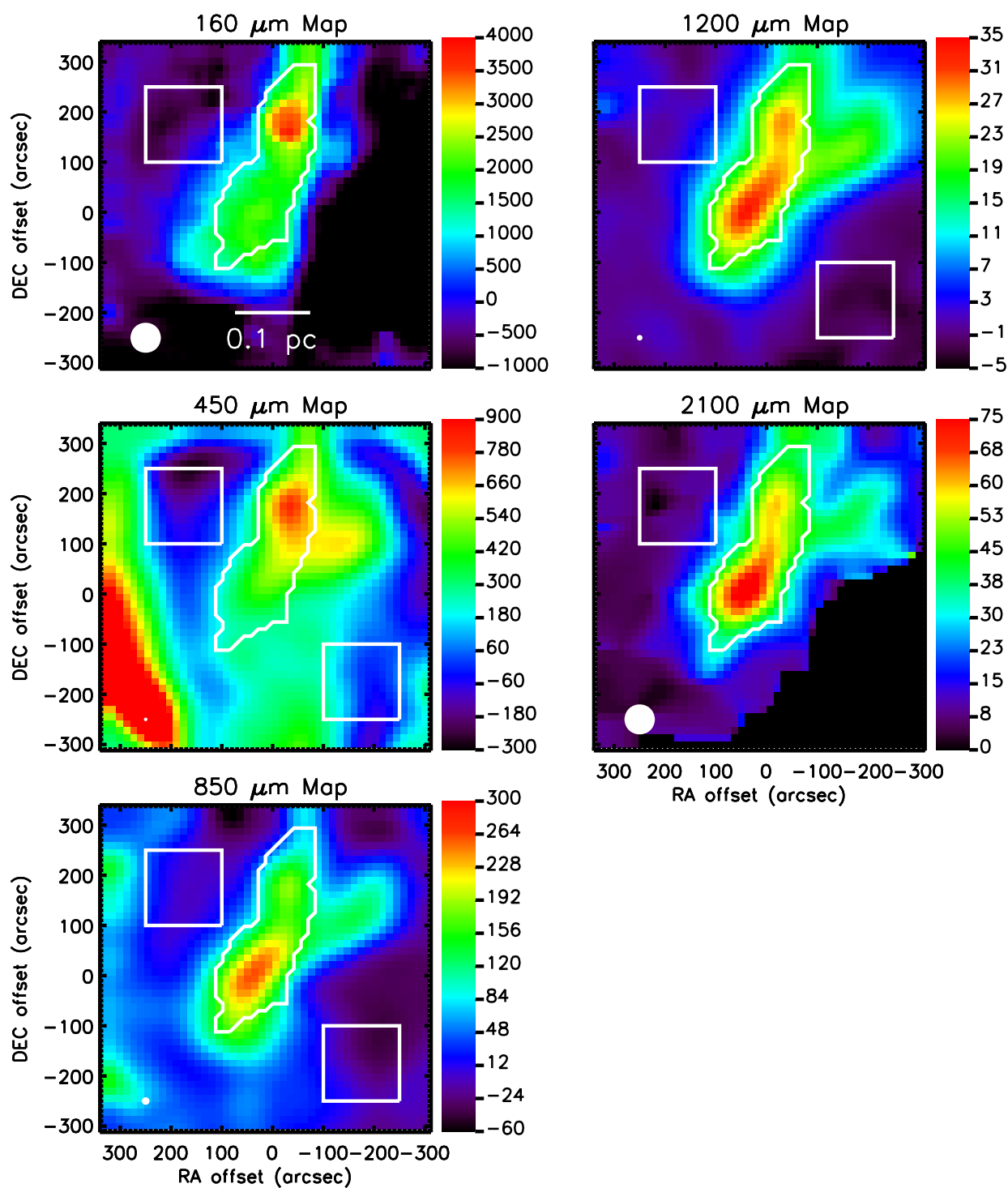

Figure 1. Flux maps of TMC-1C at 160, 450,850,1200, and $2100 \mu \mathrm{m}$, in units of mJy beam ${ }^{-1}$, smoothed with the $2100 \mu \mathrm{m}$ map's beam. The white contour shows the region that is above $3 \sigma$ in the $160,850,1200$, and $2100 \mu \mathrm{m}$ maps and above $2 \sigma$ in the $450 \mu \mathrm{m}$ map, and is the region used for determining the dust properties. The white boxes show the regions over which the noise in the maps was calculated. The beam size of each map is shown in the bottom left corners of each panel. (A color version of this figure is available in the online journal.)

Table 1

TMC-1C Fluxes

\begin{tabular}{lrcc}
\hline \hline $\begin{array}{c}\text { Nominal Wavelength } \\
(\mu \mathrm{m})\end{array}$ & $\begin{array}{r}\text { Reference Wavelength } \\
(\mu \mathrm{m})\end{array}$ & $\begin{array}{c}\text { Average Flux } \\
\left(\mathrm{Jy} \mathrm{beam}^{-1}\right)^{\mathrm{a}}\end{array}$ & Flux Uncertainty \\
\hline 160 & 159 & 2.0 & $20 \%$ \\
450 & 456 & 5.6 & $50 \%$ \\
850 & 856 & 1.1 & $20 \%$ \\
1200 & 1090 & 0.70 & $10 \%$ \\
2100 & 2103 & 0.050 & $20 \%$ \\
\hline
\end{tabular}

Note. ${ }^{a}$ In a $60^{\prime \prime}$ Gaussian beam.

(given by Equations (1)-(3)) to the five fluxes (at 160, 450, 850, 1200 , and $2100 \mu \mathrm{m}$ ) at each position. Although the resolutions of the maps at 450, 850, and $1200 \mu \mathrm{m}$ are fairly similar (from $\left.7^{\prime \prime} 5-14^{\prime \prime}\right)$, the $60^{\prime \prime}$ resolution of the 160 and $2100 \mu \mathrm{m}$ maps is not a good match to the others. Here we handle the mismatched beam sizes by making an average SED from the five flux maps to derive the best-fit dust properties $\left(T_{d}, N_{\mathrm{H}_{2}}\right.$, and $\left.\beta\right)$.
We first smooth the 450, 850, and $1200 \mu \mathrm{m}$ maps to the resolution of the 160 and $2100 \mu$ m maps by convolution with a two-dimensional Gaussian beam. We then calculate the average flux in those pixels brighter than $3 \sigma$ in the $160,850,1200$, and $2100 \mu \mathrm{m}$ maps, and brighter than $2 \sigma$ in the $450 \mu \mathrm{m}$ map (which is noisier than the other maps). The area with significant emission in all five maps is shown in Figure 1. The noise level in 

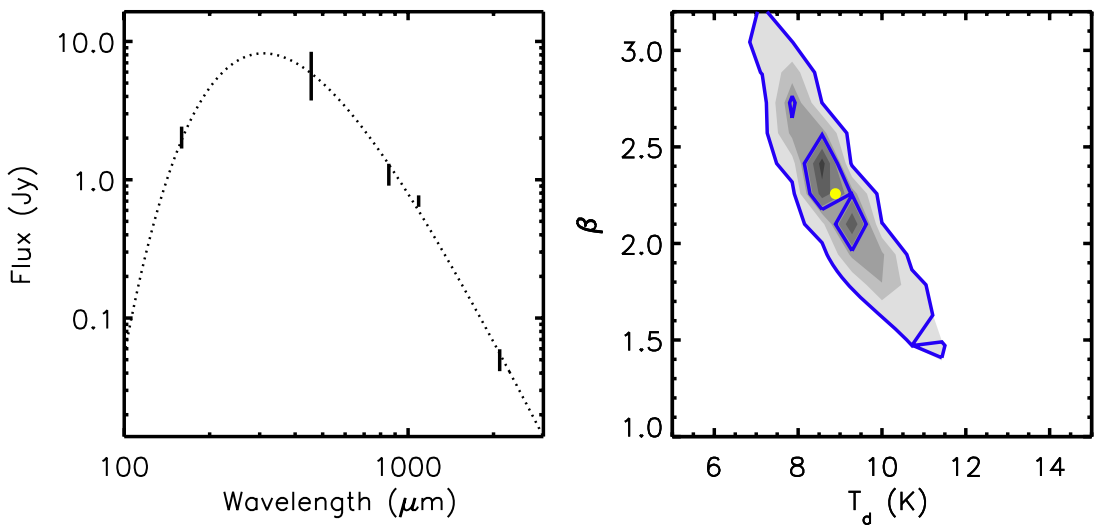

Figure 2. Left: the fluxes of TMC-1C at 160, 450, 850, 1200, and $2100 \mu \mathrm{m}$, in a $60^{\prime \prime}$ Gaussian beam, averaged over the high signal-to-noise portion of the maps, shown with $1 \sigma$ error bars. The dotted line shows the best-fit modified blackbody spectrum, which has column density $N_{\mathrm{H}_{2}}=10.2 \times 10^{21}$, dust temperature $T_{d}=8.9$, and emissivity spectral index $\beta=2.3$. See Section 3.2 for details. Right: filled contours show the density of $T_{d}-\beta$ values derived in the Monte Carlo simulation of the average TMC-1C fluxes, with the open blue contours containing 50\% and $95 \%$ of all points. The yellow circle shows the position of the median dust temperature and emissivity spectral index, used to calculate the SED in the left-hand side of this figure. The fluxes and uncertainties are given in Table 1.

(A color version of this figure is available in the online journal.)

the maps is determined in regions off-source but near TMC-1C (see Figure 1). We then used a $\chi^{2}$ minimization routine to fit the five measured fluxes with a modified blackbody SED, finding the values of $T_{d}, N_{\mathrm{H}_{2}}$, and $\beta$.

In order to determine how noise and calibration uncertainties affect the accuracy of the derived dust properties, we run a simple Monte Carlo simulation. In each trial, Gaussian random noise of the amount observed is added to each flux map, and then each map is scaled by a multiplicative factor $f=1.0+\delta$, where $\delta$ is randomly chosen from a normal distribution of mean zero and standard deviation equal to the absolute calibration uncertainty in each map. Each map is modified by a different scale factor $f$, and this process was repeated 5000 times. We find that the best-fit dust properties for the average SED over TMC-1C are $\beta=2.3 \pm 0.4, T_{d}=8.9 \pm 1.0$, and $N_{\mathrm{H}_{2}}=(10.2 \pm 2.1) \times 10^{21}$. The observed fluxes and best-fit SED are plotted in Figure 2, and the fluxes and uncertainties are given in Table 1.

A fundamental flaw in this analysis is that Equations (1)(3) are only accurate for isothermal dust characterized by a single emissivity spectral index, but TMC-1C is known to be warmer at its edges than at the center (Schnee et al. 2007b), and it is certainly possible that $\beta$ also changes within the starless core. As shown by Shetty et al. (2009a), the incorrect assumption of isothermal dust drives an anticorrelation between the derived dust temperature and emissivity spectral index. A spurious anticorrelation between $T_{d}$ and $\beta$ may also arise due to noise in the emission maps (Shetty et al. 2009a, 2009b). An anticorrelation between $\beta$ and $T_{d}$ is seen in the Monte Carlo simulation we used to derive the dust properties, as shown in Figure 2. In the following section, we attempt to mitigate the effects of variations in the dust properties on our analysis.

\subsection{Maps}

The dust properties $\left(T_{d}, N_{\mathrm{H}_{2}}\right.$, and $\left.\beta\right)$ in TMC-1C may vary both in the plane of the sky and along the line of sight. The lineof-sight variations in the dust properties cannot be determined without knowledge of the geometry of the core, but variations in the plane of the sky are more tractable. Here we make maps of TMC-1C, first of the temperature and column density while holding the emissivity spectral index constant, and then of all three quantities.

\subsubsection{Maps with Constant $\beta$}

We first regrid the 450,850 , and $1200 \mu \mathrm{m}$ maps to the resolution of the $850 \mu \mathrm{m}$ map, 14". We then determine the noise in the maps in the same regions selected in Section 3.2 and calculate the dust temperature and column density in those pixels brighter than $3 \sigma$ in the 850 and $1200 \mu \mathrm{m}$ maps and brighter than $2 \sigma$ in the $450 \mu \mathrm{m}$ map, using Equations (1)-(3) for an assumed value of the emissivity spectral index, as was done in Schnee et al. (2007b). Temperature and column density maps are made for each value of the emissivity spectral index in the range $1.0 \leqslant \beta \leqslant 4.0$ in steps of 0.1 . To account for the effects of noise and calibration uncertainties, we use the same Monte Carlo technique described in Section 3.2 to add Gaussian random noise to each pixel and adjust each map by a multiplicative scale factor, and this process is repeated 100 times for each value of $\beta$.

To determine which value of $\beta$ is most appropriate for TMC$1 \mathrm{C}$, we then use the derived $T_{d}$ and $N_{H}$ maps to predict the fluxes at 160, 450, 850, 1200, and $2100 \mu \mathrm{m}$. The predicted 160 and $2100 \mu \mathrm{m}$ fluxes are convolved with a $60^{\prime \prime}$ Gaussian beam to match the resolution of the observed maps. We find that the 160 and $2100 \mu \mathrm{m}$ maps are best reproduced by an emissivity spectral index of $\beta=2.2 \pm 0.6$. The observed, predicted and residual flux maps for $\beta=2.2$ are shown in Figure 3, and the derived temperature and column density maps are shown in Figure 4. The column density is expressed in units of $A_{V}$, using the conversion factor $N_{\mathrm{H}_{2}}=9.4 \times 10^{20} \mathrm{~cm}^{-2}\left(A_{V} \mathrm{mag}^{-1}\right.$; Bohlin et al. 1978).

\subsubsection{Maps with Variable $\beta$}

In Section 3.3.1, we assumed that $\beta$ is constant across TMC$1 \mathrm{C}$ to make $14^{\prime \prime}$ maps of the dust temperature and column density in TMC-1C. Here we make two-dimensional maps of the dust temperature, column density, and emissivity spectral index in TMC-1C, with the tradeoff that the resultant maps have coarser resolution $\left(60^{\prime \prime}\right)$.

We first regrid the 450,850 , and $1200 \mu \mathrm{m}$ maps to the $60^{\prime \prime}$ resolution of the 160 and $2100 \mu \mathrm{m}$ maps. For each pixel brighter than $3 \sigma$, we then calculate $T_{d}, N_{\mathrm{H}_{2}}$, and $\beta$ using Equations (1)(3). To estimate the uncertainties in this method due to noise and absolute calibration errors, we used the same Monte Carlo techniques as in Sections 3.2 and 3.3.1 with 1000 trials. In each 

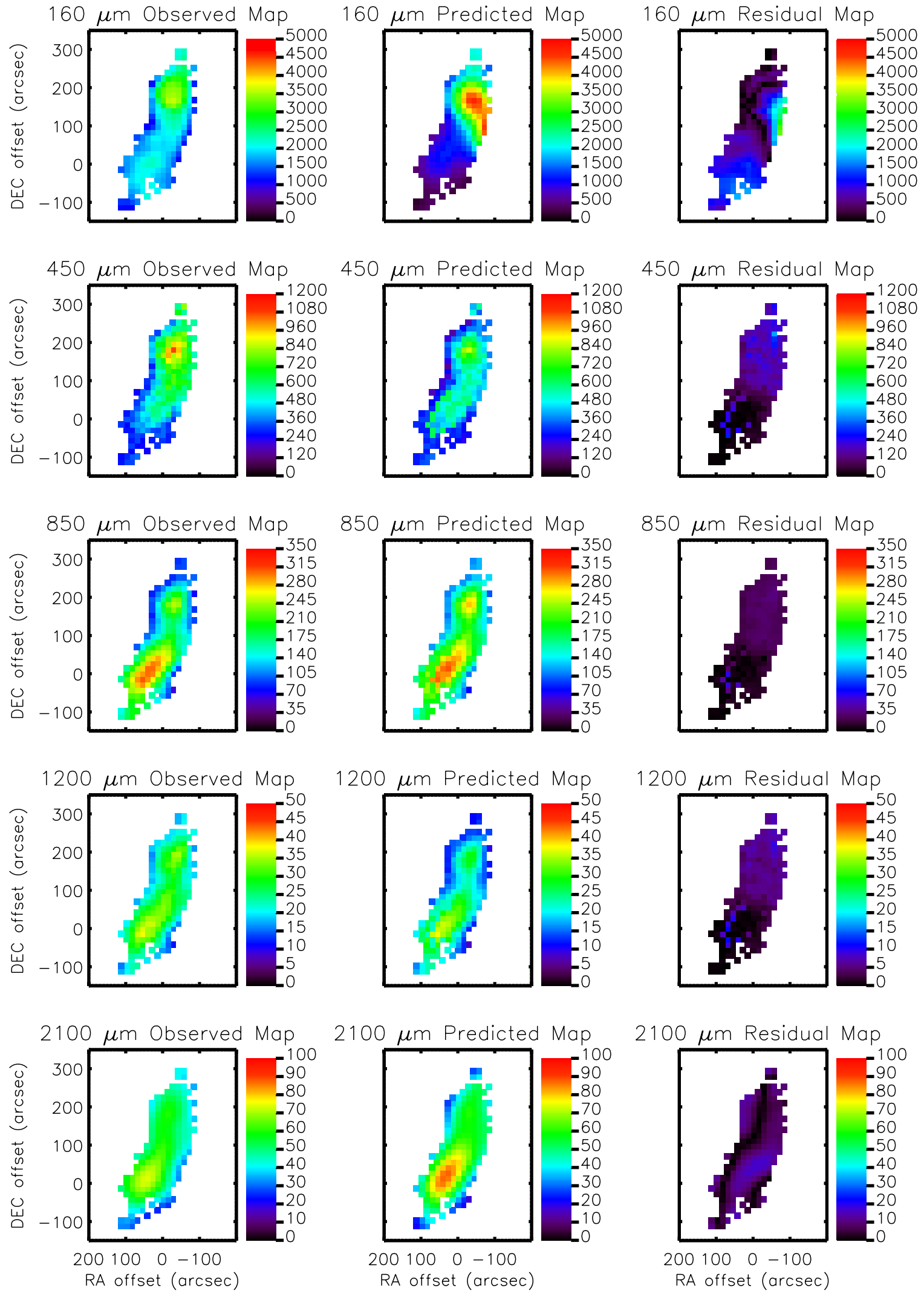

Figure 3. Left column: the observed maps of TMC-1C at 160, 450, 850, 1200, and $2100 \mu \mathrm{m}$, shown in units of mJy beam ${ }^{-1}$. Center column: the predicted maps of TMC-1C, based on the derived temperature and column density maps (see Figure 4), with $\beta=2.2$. Right column: the absolute value of the residuals. See Section 3.3.1 for details.

(A color version of this figure is available in the online journal.)

trial there were about 15 pixels with sufficiently high signal to noise to calculate the dust properties, and within the maps there is a clear anticorrelation between $T_{d}$ and $\beta$, as shown in Figure 4. The median emissivity spectral index and dust 

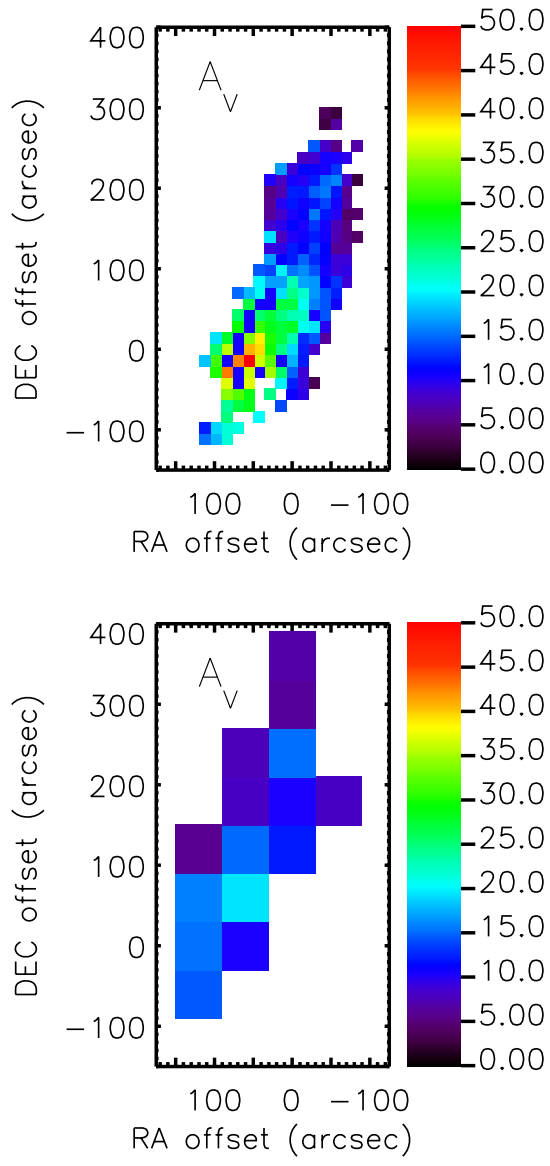
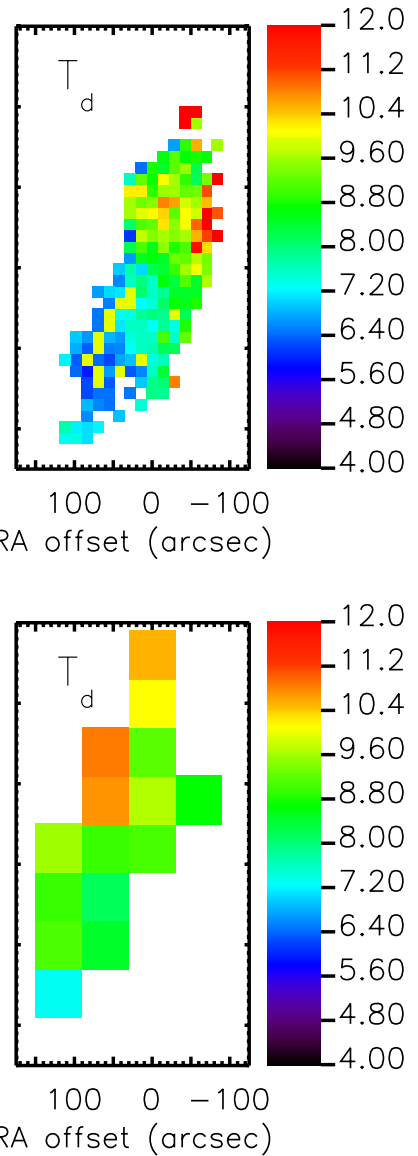

RA offset (arcsec)
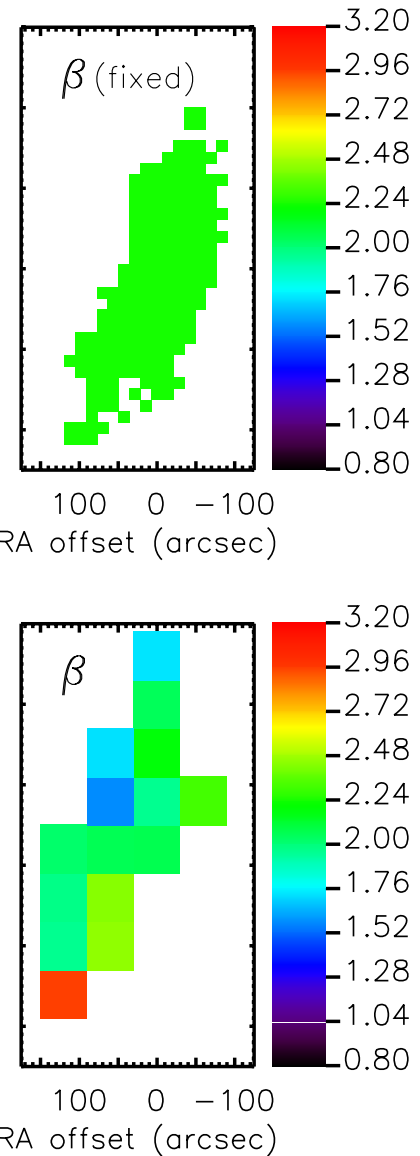

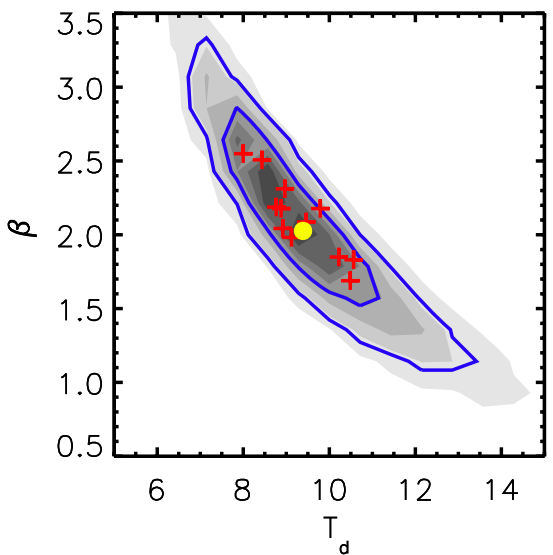

Figure 4. Top row: column density and dust temperature maps, expressed in units of $A_{V}$ and $\mathrm{K}$, derived from $14^{\prime \prime}$ maps of TMC-1C at 450,850 , and $1200 \mu \mathrm{m}$ using $\beta=2.2$. See Section 3.3.1 for details. Center row: column density, dust temperature, and emissivity spectral index maps of TMC-1C, derived from $60^{\prime \prime}$ maps at 160 , $450,850,1200$, and $2100 \mu \mathrm{m}$. See Section 3.3.2 for details. Bottom row: filled contours show the density of $T_{d}-\beta$ values, with the open blue contours containing $50 \%$ and $95 \%$ of all points. The yellow circle shows the position of the median dust temperature $\left(T_{d}=9.4\right)$ and emissivity spectral index $(\beta=2.0)$. The red crosses show the values of $\beta$ and $T_{d}$ derived from each independent position in the $60^{\prime \prime}$ flux density maps with high signal to noise.

(A color version of this figure is available in the online journal.)

temperature derived from the high signal-to-noise points in all trials are $\beta=2.0 \pm 0.6$ and $T_{d}=9.4 \pm 1.9$, similar to that derived from the composite SED of TMC-1C in Section 3.2.

\section{DISCUSSION}

In Section 3.3.2, we found that there is an anticorrelation between the dust temperature and emissivity spectral index in the two-dimensional maps of TMC-1C. Although this phenomenon has been reported in other star-forming regions (Dupac et al.
2003) and in other galaxies (Yang \& Phillips 2007), this is not a known feature of starless cores. The assumption that the temperature along each line of sight is constant, even though the temperature profile in TMC-1C is very likely to be colder at higher densities, can lead to the mistaken conclusion that there is an anticorrelation between $T_{d}$ and $\beta$ (Shetty et al. 2009 a , 2009b). Noise in the dust emission maps can also drive a spurious $T_{d}-\beta$ anticorrelation. Here we investigate whether the dust temperature and emissivity spectral index are truly anticorrelated, and conclude that this is not necessarily the case. 

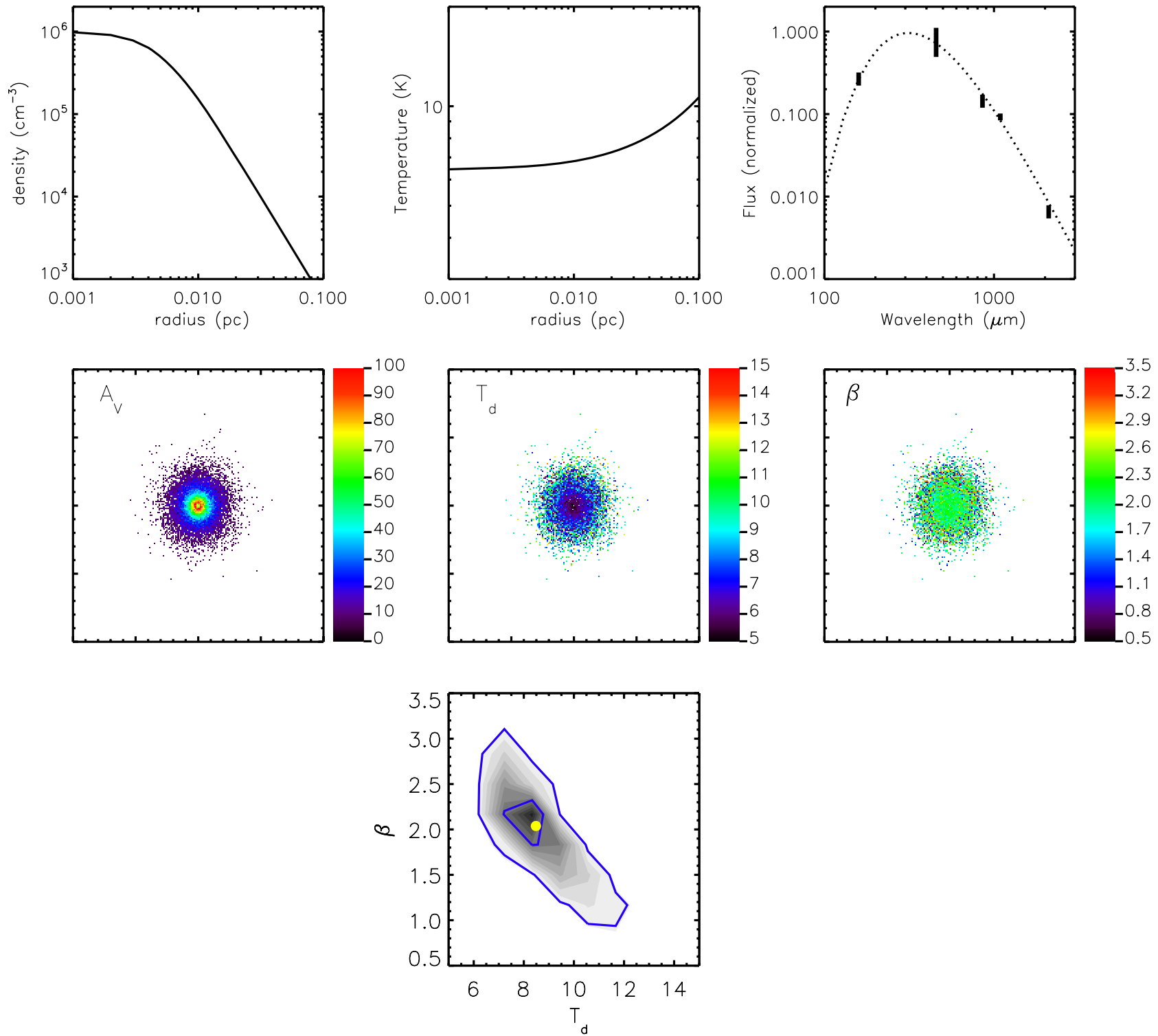

Figure 5. Top left and center: density and temperature profiles of a spherical model of a starless core. Top right: the SED of the model starless core with $\beta=2.2$, normalized such that the SED peak is at unity. The TMC-1C observations are also plotted for reference, and scaled by a common factor such that the $450 \mu \mathrm{m}$ flux from TMC-1C is equal to that of the model core. Center row: column density, temperature, and emissivity spectral index maps that would be derived from the noisy maps of the model starless core. Bottom row: filled contours show the density of $T_{d}-\beta$ values, with the open blue contours containing $50 \%$ and $95 \%$ of all points. The yellow circle shows the position of the median dust temperature and emissivity spectral index. The anticorrelation between $T_{d}$ and $\beta$ is similar to that observed in TMC-1C (see Figure 4).

(A color version of this figure is available in the online journal.)

We then determine what constant value of $\beta$ best characterizes TMC-1C.

\subsection{Testing the $T_{d}-\beta$ Anticorrelation}

To determine the effects of temperature variations along the line of sight and noise on the dust properties derived from flux maps at 160, 450, 850, 1200, and $2100 \mu \mathrm{m}$, we consider a spherical model of a starless core with plausible properties. The density in the core is given by

$$
n(r)=\frac{n_{0}}{1+\left(r / r_{0}\right)^{2.5}},
$$

where $r$ is the radius, $n_{0}=10^{6} \mathrm{~cm}^{-3}$, and $r_{0}=0.005 \mathrm{pc}$. The temperature in the core is given by

$$
T_{d}(r)=T_{0}\left(1+r / r_{1}\right) \text {, }
$$

where $T_{0}=6.4 \mathrm{~K}$ and $r_{1}=0.15 \mathrm{pc}$. The emissivity spectral index is constant in the core at a value $\beta=2.2$. This model core is colder and denser at the center, as expected for an externally heated starless core. We do not claim that these are the temperature and density profiles for TMC-1C, but there is a general agreement between the shape of the model SED and that of TMC-1C. The density and temperature profiles are shown in Figure 5, as is the total emergent SED.

For each line of sight passing through the model core, we determine the volume of intersection between a cylinder and each spherical shell of the core. Given the density and temperature distributions from Equations (5) and (6), the flux from each layer is given by

$$
S_{v}=\frac{B_{v}\left(T_{d}\right) \kappa_{\nu} n_{r} V_{r}}{d^{2}},
$$


where $V_{r}$ is the volume of intersection and $d$ is the distance to the core. The two-dimensional flux distribution is calculated by summing the flux from each layer along each line of sight. For the sake of convenience, the total flux density of the model core, summed over all radii, is scaled by a common factor such that the peak of the SED has a value of unity.

We "observe" the model core by deriving two-dimensional flux maps at 160, 450, 850,1200, and $2100 \mu \mathrm{m}$ and adding Gaussian random noise equal to $5 \%$ of the peak flux in each map. Using Equations (1)-(3), we then derive two-dimensional maps of the column density, dust temperature, and emissivity spectral index, assuming that these quantities are constant along the line of sight (as was done in Section 3.3.2). The derived column density map peaks at the center of the core, where the temperature is seen to decrease, as one would expect from the input density and temperature profiles (see Figure 5). The derived emissivity spectral index map covers a wide range of values, mostly between $1.0 \leqslant \beta \leqslant 3.5$, even though the input emissivity spectral index was held constant. The median-derived emissivity spectral index is $2.0 \pm 0.4$, which is close to the input value of $\beta=2.2$.

Furthermore, the derived temperature and emissivity spectral index are seen to be anticorrelated. The combination of noise and temperature variations along the line of sight have been shown to drive an anticorrelation between these two quantities (Shetty et al. 2009a, 2009b), so it is not surprising that this effect is seen here. Interestingly, the portion of the $T_{d}-\beta$ parameter space covered by the model starless core overlaps significantly with that of TMC-1C (see Section 3.3.2 and Figure 5). From this, we conclude that there is no definitive evidence that the emissivity spectral index in TMC-1C varies with temperature, and that the more reliable estimates of column density and temperature will come from fitting the observed fluxes while holding the emissivity spectral index constant.

\subsection{Average Emissivity Spectral Index in TMC-1C}

Estimates of the emissivity spectral index in TMC-1C derived from its average SED (see Section 3.2) and from twodimensional maps using the 450,850 , and $1200 \mu \mathrm{m}$ maps to predict the lower resolution 160 and $2100 \mu \mathrm{m}$ maps (see Section 3.3.1), $\beta=2.3 \pm 0.4$ and $\beta=2.2 \pm 0.6$, are in good agreement with each other. Given that an analysis of the flux maps from an idealized core, with line-of-sight temperature variations and noise, gives the correct average emissivity spectral index (within errors), we suggest that the value of the emissivity spectral index in TMC-1C is well represented by $\beta \simeq 2.2 \pm 0.5$. This is consistent with the emissivity spectral index in the diffuse ISM of $\beta=2$ (Draine et al. 2007; Draine \& Lee 1984) and with the emissivity spectral index measured in the starless core L1498 ( $\beta=2.44 \pm 0.62$; Shirley et al. 2005). However, due to the large uncertainty in $\beta$ in this study, we cannot rule out the possibility that the emissivity spectral index in TMC-1C could also be intermediate between the shallower SEDs seen in circumstellar disks and the SED of the ISM.

When deriving the dust temperature and mass of prestellar cores from sparsely sampled SEDs, it is common to assume that the emissivity spectral index of the dust is equal to 2 (e.g., Kirk et al. 2007; Simpson et al. 2008). Observations of L1498 (Shirley et al. 2005) and our observations of TMC-1C are consistent with $\beta=2$, though the best-fit values of the emissivity spectral index in both cores are slightly steeper, which would result in lower temperatures and higher masses. However, with so few measurements of $\beta$ in starless cores, there is not sufficient information with which to re-examine the dust properties in previously published studies. Upcoming surveys of the Gould Belt molecular clouds using Herschel $l^{9}$ and $S C U B A-2^{10}$ promise to make statistically significant measurements of the emissivity spectral index in starless cores.

\section{SUMMARY}

In this paper, we have analyzed the dust emission at 160 , $450,850,1200$, and $2100 \mu \mathrm{m}$ from TMC-1C. Our previous studies of this starless core have mapped the dust temperature and column density. Here, using new 160 and $2100 \mu \mathrm{m}$ maps and an improved reduction of the 450 and $850 \mu \mathrm{m}$ maps, we are able to estimate the emissivity spectral index and make an improved analysis of the temperature and column density in TMC-1C.

We find that the spectral index of the dust emission, as calculated by a variety of related methods, is in the range $1.7 \leqslant \beta \leqslant 2.7$. This is consistent with the value of the emissivity spectral index often assumed in studies of starless cores, and is also consistent with laboratory measurements and observations of the behavior of dust grains at low temperatures $\left(T_{d} \leqslant 10 \mathrm{~K}\right)$. However, there is still a relatively large uncertainty in the emissivity spectral index.

Using the new estimate of the emissivity spectral index in TMC-1C $(\beta=2.2)$, we construct temperature and column density maps, which reach values of $T_{d} \simeq 7 \mathrm{~K}$ and $A_{V} \simeq 50$ at the position of the dust peak, as shown in Figure 4. We find no conclusive evidence that $\beta$ varies within the core, though line-of-sight temperature variations and noise in the flux maps leave open the possibility that the emissivity spectral index is anticorrelated with dust temperature.

We thank our referee, Yancy Shirley, for comments that improved the clarity of this paper. S.S. acknowledges support from the Owens Valley Radio Observatory, which is supported by the National Science Foundation through grant AST 0540399. J.S. was partially supported by a NASA Postdoctoral Program Fellowship. Support was provided to M.E. by NASA through the Spitzer Space Telescope Fellowship Program. The JCMT is operated by the Joint Astronomy Centre on behalf of the Particle Physics and Astronomy Research Council of the United Kingdom, the Netherlands Organisation for Scientific Research, and the National Research Council of Canada. IRAM is supported by INSU/CNRS (France), MPG (Germany), and IGN (Spain). The CSO is supported by the NSF fund under contract AST 02-29008.

\section{REFERENCES}

Aannestad, P. A. 1975, ApJ, 200, 30

Agladze, N. I., Sievers, A. J., Jones, S. A., Burlitch, J. M., \& Beckwith, S. V. W. 1996, ApJ, 462, 1026

Beckwith, S. V. W., \& Sargent, A. I. 1991, ApJ, 381, 250

Bianchi, S., Gonçalves, J., Albrecht, M., Caselli, P., Chini, R., Galli, D., \& Walmsley, M. 2003, A\&A, 399, L43

Bohlin, R. C., Savage, B. D., \& Drake, J. F. 1978, ApJ, 224, 132

Boudet, N., Mutschke, H., Nayral, C., Jäger, C., Bernard, J.-P., Henning, T., \& Meny, C. 2005, ApJ, 633, 272

Chakrabarti, S., \& McKee, C. F. 2005, ApJ, 631, 792

Di Francesco, J., Johnstone, D., Kirk, H., MacKenzie, T., \& Ledwosinska, E. 2008, ApJS, 175, 277

Draine, B. T., \& Lee, H. M. 1984, ApJ, 285, 89

\footnotetext{
9 http://starformation-herschel.iap.fr/gouldbelt

10 http://www.jach.hawaii.edu/JCMT/surveys/gb/
} 
Draine, B. T., et al. 2007, ApJ, 663, 866

Dupac, X., et al. 2003, A\&A, 404, L11

Emerson, D. T., Klein, U., \& Haslam, C. G. T. 1979, A\&A, 76, 92

Enoch, M. L., Evans, N. J., II, Sargent, A. I., Glenn, J., Rosolowsky, E., \& Myers, P. 2008, ApJ, 684, 1240

Enoch, M. L., et al. 2006, ApJ, 638, 293

Flagey, N., et al. 2009, ApJ, 701, 1450

Hatchell, J., Fuller, G. A., Richer, J. S., Harries, T. J., \& Ladd, E. F. 2007, A\&A, 468,1009

Högbom, J. A. 1974, A\&AS, 15, 417

Holland, W. S., et al. 1999, MNRAS, 303, 659

Kauffmann, J., Bertoldi, F., Bourke, T. L., Evans, N. J., II., \& Lee, C. W. 2008, A\&A, 487, 993

Keto, E., \& Caselli, P. 2008, ApJ, 683, 238

Keto, E., Rybicki, G. B., Bergin, E. A., \& Plume, R. 2004, ApJ, 613, 355

Kirk, J. M., Ward-Thompson, D., \& André, P. 2007, MNRAS, 375, 843

Kreysa, E., et al. 1999, Infrared Phys. Technol., 40, 191

Kuan, Y.-J., Mehringer, D. M., \& Snyder, L. E. 1996, ApJ, 459, 619

Lis, D. C., Serabyn, E., Keene, J., Dowell, C. D., Benford, D. J., Phillips, T. G., Hunter, T. R., \& Wang, N. 1998, ApJ, 509, 299

Mennella, V., Brucato, J. R., Colangeli, L., Palumbo, P., Rotundi, A., \& Bussoletti, E. 1998, ApJ, 496, 1058
Nutter, D., Kirk, J. M., Stamatellos, D., \& Ward-Thompson, D. 2008, MNRAS, 384,755

Ossenkopf, V., \& Henning, T. 1994, A\&A, 291, 943

Sayers, J., et al. 2009, ApJ, 690, 1597

Schnee, S., Caselli, P., Goodman, A., Arce, H. G., Ballesteros-Paredes, J., \& Kuchibhotla, K. 2007a, ApJ, 671, 1839

Schnee, S., \& Goodman, A. 2005, ApJ, 624, 254

Schnee, S., Kauffmann, J., Goodman, A., \& Bertoldi, F. 2007b, ApJ, 657, 838

Schnee, S., Rosolowsky, E., Foster, J., Enoch, M., \& Sargent, A. 2009, ApJ, 691,1754

Schwarz, U. J. 1978, A\&A, 65, 345

Shetty, R., Kauffmann, J., Schnee, S., Goodman, A. A., \& Ercolano, B. 2009a, ApJ, 696, 2234

Shetty, R., Kauffmann, J., Schnee, S., \& Goodman, A. A. 2009b, ApJ, 696, 676

Shirley, Y. L., Nordhaus, M. K., Grcevich, J. M., Evans, N. J., II, Rawlings, J. M. C., \& Tatematsu, K. 2005, ApJ, 632, 982

Simpson, R. J., Nutter, D., \& Ward-Thompson, D. 2008, MNRAS, 391, 205

Stansberry, J. A., et al. 2007, PASP, 119, 1038

Terebey, S., et al. 2009, ApJ, 696, 1918

Torres, R. M., Loinard, L., Mioduszewski, A. J., \& Rodríguez, L. F. 2009, ApJ, 698,242

Weingartner, J. C., \& Draine, B. T. 2003, ApJ, 589, 289

Yang, M., \& Phillips, T. 2007, ApJ, 662, 284 